\title{
Therapeutic Class III molar occlusion
}

\author{
Camille PHILIP-ALLIEZ, Michel LE GALL, \\ Danielle DEROZE, Jean-Daniel ORTHLIEB, \\ Pierre CANAL
}

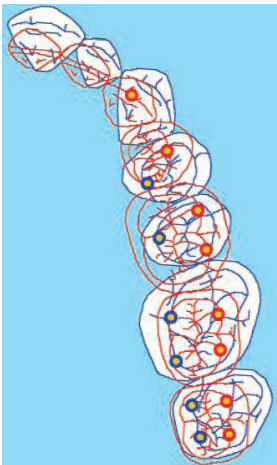

\begin{abstract}
The objective of this work is to study the static and dynamic occlusion of dentitions in a Class III molar relationship to determine what might be the indications for treatment that would leave buccal segments in this position, and to evaluate the occlusal adaptions that would be needed to make it functionally acceptable.
\end{abstract}

\section{KEYWORDS}

Occlusion

Class III

Occlusal equilibration. 


\section{1 - INTRODUCTION}

A primary therapeutic objective of orthodontic treatment is to obtain a Class I canine and molar relationship and to provide functional anterior guidance.

In some specific cases, orthodontists have concluded that a "therapeutic" Class II molar relationship, associated with a Class I canine relation and harmony with well equilibrated incisal segments is acceptable so long as the upper molars are positioned in a manner different from those in a Class I relationship. This "therapeutic" Class II relationship has been considered suitable because, as a part of a well equilibrated dentition it is deemed not to be traumatogenic.

We are suggesting the hypothesis that orthodontists should consider bestowing the same acceptable status on a "therapeutic" Class III relationship. We shall attempt to validate

\section{2 - INDICATIONS}

In cases of congenitally absent lower second premolars, well formed and complete maxillary arch, and a flat profile, a treatment plan calling for no compensating maxillary extractions and mandibular space closure by mesial movement of the buccal segments might be appropriate especially where economic considerations are a factor.

Similar treatment plans may be appropriate for cases of excess mesial positioning of lower molars where moving them distally might pose serious technique problems or risk this hypothesis and we shall try to establish the criteria for the rare indications for treatment that would result in such a termination.

We shall also endeavor to determine what type of static and dynamic occlusions should be present when first molars are left in a therapeutic Class III relationship.

However, it should be clear that a Class III molar relationship, even when associated with a Class I canine position and well functioning incisors blocks, should not be considered in itself an orthodontic objective but, instead, an occlusal compromise.

In addition, as we shall postulate later in this paper, it is possible to ask the question whether a finished result in therapeutic Class III, despite the reservations we might have about it, can be considered a "lesser evil" in some situations. causing an unacceptable increase in vertical dimension.

Similar considerations may apply in cases of mild skeletal Class III with no dento-alveolar compensation that shows a mandibular arch with anterior crowding while the teeth in maxillary arch are perfectly aligned. By extracting two lower premolars or one lower incisor the orthodontist can resolve the problem of the crowding of the mandibular anteriors and their relationship to their maxillary antagonists without making any demands on the 
satisfactory upper arch. The alternative procedure of extracting the upper second premolars to mesialize the first molars and obtain a Class I molar relationship would risk "dishing in" the face.

A similar problem can arise in Class II skeletal cases when retracting the mandibular incisors to obtain sufficient overjet to accommodate surgical advancement of the mandible requires the extraction of two lower premolars. Traditional treatment plans would call for compensatory maxillary extrac-

\section{3 - CONTRA-INDICATIONS}

Contra-indications for orthodontic treatment leaving molars in Class III relationship are essentially those of a Class III skeletal type that require surgical intervention. In these cases, where there is often a problem of vertical dimension, the skeletal discrepancy is so great that no dento-alveolar compensation can be accomplished. Most often surgery in both jaws is required.

And if the lower third molars are congenitally absent, there is added

\section{4 - CURRENT CONCEPTS OF OCCLUSION}

Clark and Evans $^{1}$ reviewed the literature searching for criteria that could define ideal functional occlusion, but were unable to find any clear and well accepted benchmarks, writing, "no pertinent definition of the ideal occlusion can be established in a conclusive fashion." At best, they discerned a convergence of ideas of tions in order to achieve a Class I molar relationship even if the upper arch is well aligned. Are such extractions always necessary?

If the end-of-treatment Class III dentition can be well enough equilibrated to eliminate all occlusal trauma, can we consider it to be "therapeutic" provided that the canines are in Class I and the incisors are in good function? We believe that indications for such a solution exist even though they are rare.

reason to presume that a final "therapeutic" Class III molar relationship would be imprudent.

In cases of congenital absence of lower second premolars, closing the spaces by a planned mesial movement of the molar teeth is a tempting prospect, but orthodontists must evaluate the risk of its adversely affecting the appearance of patients with flat or concave profiles when the inevitable reciprocal distal movement of the incisal block occurs.

authors about competing principles for the achievement of good occlusion:

- both left and right segments of the upper and lower arches should be in contact in centric relation.

- there should be less than $1 \mathrm{~mm}$ difference between centric relation and maximum intercuspation. 
- contacts in lateral excursion should occur only on the working side, either between canines, in canine protected occlusion, or between two pairs of laterally adjacent teeth in the buccal segments, in group function, with no contact on the non-working side.

\section{5 - CRITERIA FOR FINE DETAILING OF OCCLUSION}

\section{5 - 1 - In Angle Class I normal occlusion}

Because of the nature of the intercuspation between the two arches, the teeth of the lower arch are a halfcusp in advance of the upper teeth, all teeth occluding with two antagonists except for the mandibular central incisors and the maxillary third molars (fig.1 and 2).

\section{5 - 2 - In therapeutic Class III molar occlusion}

The over-all decrease in the number of contact points between the two arches and their less favorable ar-

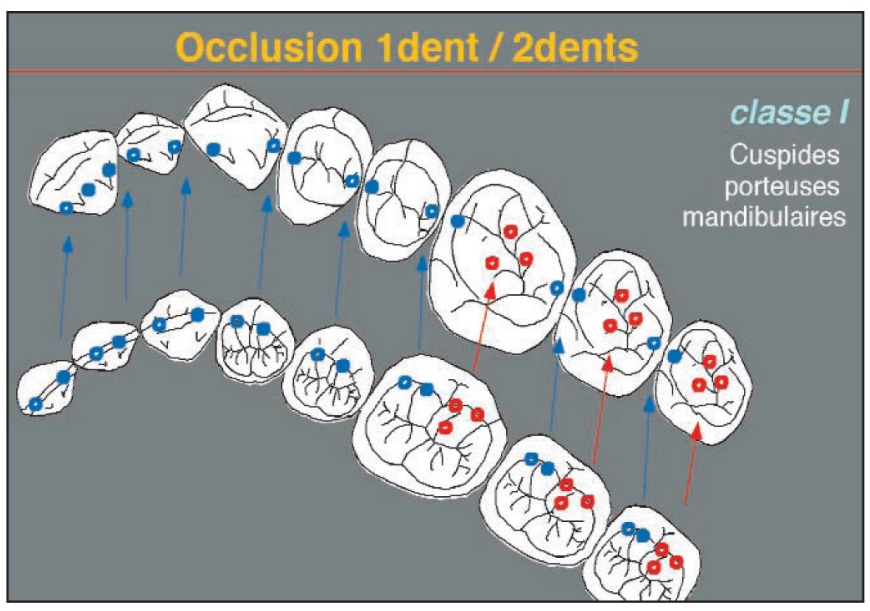

Figure 1

Occlusion 1 tooth with 2 teeth: mandibular working cusps. After Orthlieb3. rangement weakens the stability of a therapeutic Class III occlusion. In it the Class I canine relationship is preserved but the Class III molar positioning promotes a tooth on tooth interarch relationship for the maxillary second premolars and the mandibular first molars (fig. 3 and 4).

\section{5 - 2 - 1 - In the maxilla}

\section{- Static occlusion}

The palatal cusp of the maxillary second premolar should, in occlusion, lie in the central fossa of the mandibular first molar. But the mesio-palatal cusp of the first maxillary molar, in occlusion, can scarcely fit in the mesial fossa of the second mandibular molar.

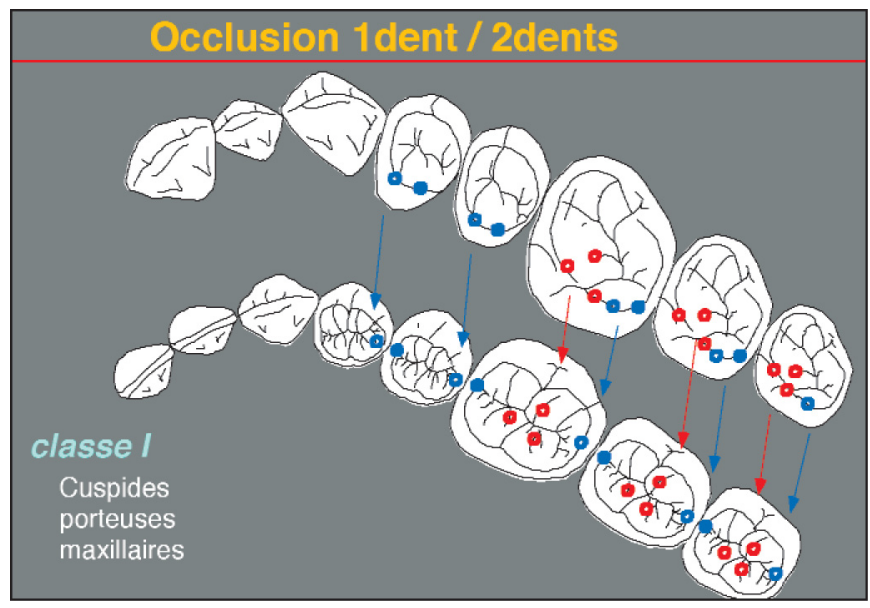

Figure 2

Occlusion 1 tooth with 2 teeth: maxillary working cusps After Orthlieb3 


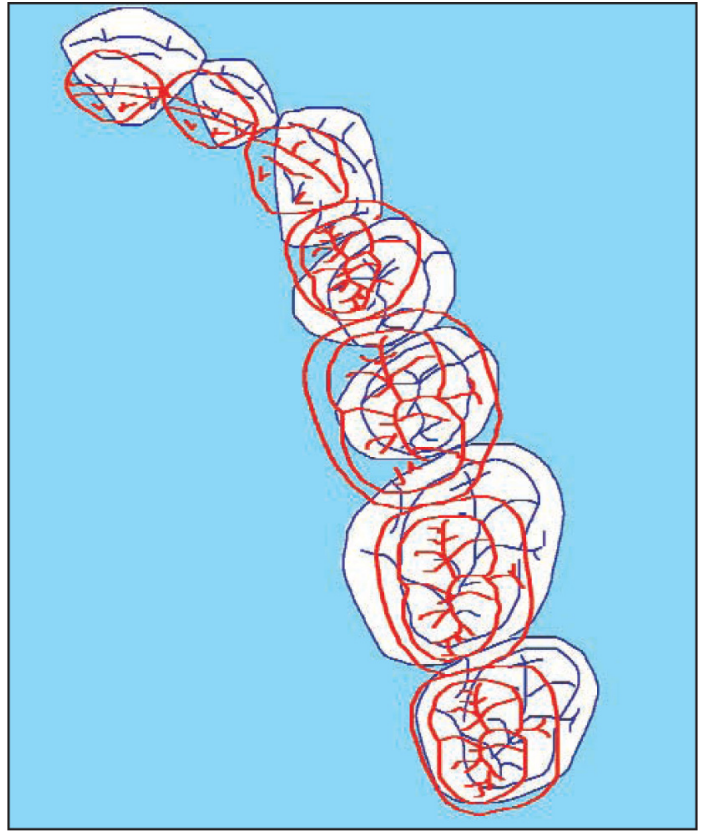

Figure 3

Horizontal view of teeth left half arches in therapeutic Class III molar occlusion. After Orthlieb3

\section{- Dynamic occlusion}

- On the working side in lateral excursion: The mesio-palatal cusp of the maxillary first molar tends to be blocked by the mesio-buccal cusp of the second mandibular molar.

- On the non-working side in lateral excursion:

The mesio-palatal cusp of the first maxillary molars buts up against the mesiolingual cusp of the second mandibular molar.

\section{5 - 2 - 2 - In the mandible}

\section{- Static occlusion}

The buccal cusp of the second mandibular premolar makes contact with the embrasure between maxillary canine and first premolar. This posi-

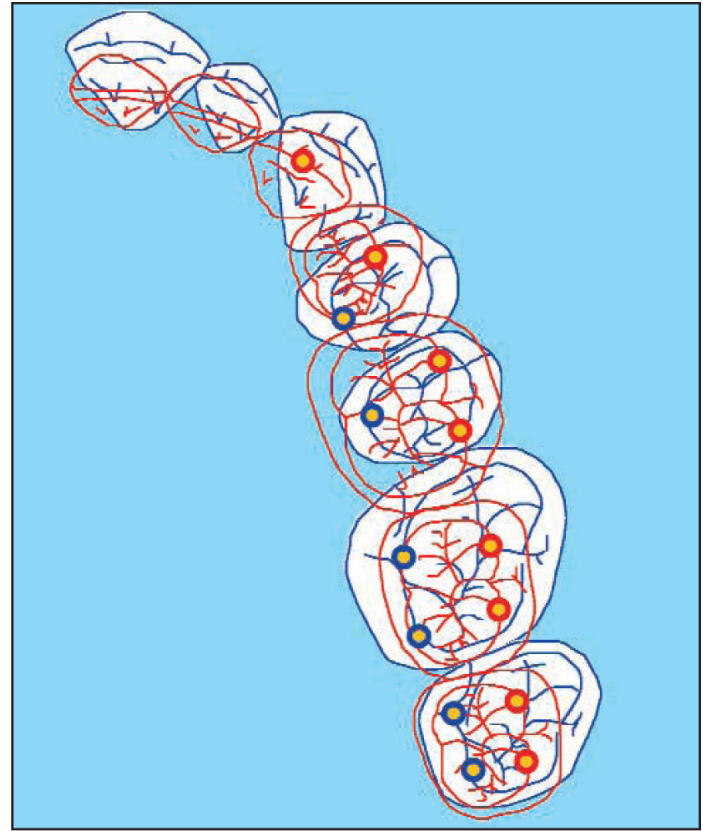

Figure 4

Occlusal contacts in therapeutic Class III molar relationship. After Orthlieb3.

tioning presents no problems when the teeth of the two arches are in occlusion or are functioning.

The mesio-buccal cusp of the mandibular first molar lodges in the opposing upper embrasure between the second maxillary premolar and the maxillary first molar.

\section{-Dynamic occlusion}

- On the working side in lateral excursion:

In lateral movements, the distal palatal surface of the palatal cusp of the upper second premolar makes a hard contact with the mesio-buccal cusp of the lower second premolar.

- In lateral movements on the nonworking side, the distal surface of the mesio-buccal cusp of the lower first 
molar will encounter the internal mesial surface of the buccal cusp of the upper second premolar.

- In protrusive movement.

The details of an adjustment of a Class III occlusion do not interfere with protrusive movements. The teeth routinely come out of occlusal contact, in part as a result of the orthodontist's regulating the components of tooth to tooth articulation: orientation of the occlusal plane, the curve of Spee, cusp height, and incisal slope to harmonize its function with condylar slope.

To assure all these aspects of correct function are in place, orthodontists must finish treatment with an occlusal equilibration that will stabilize a therapeutic Class III molar relationship.

\section{6 - OCCLUSAL EQUILIBRATION}

\section{6 - 1 - In the area of the maxillary first molar}

The orthodontist should deepen the central fossa of the upper first molar. (fig. 5) and re-shape the enamel bridge (fig. 6) so that the mesio-buccal cusp of the lower second molar can adapt itself. It is advisable to apply buccal root torque to the upper first molars to compensate for the decrease in mandibular width and thereby eliminate occlusal interferences (fig. 7).

By slightly rotating the upper first molars mesially, orthodontists can improve their occlusion with the lower second molars. So, as in regular Class II therapy, they should not use brackets with toe-in incorporated in them. In order to determine how much molars that vary in size from patient to patient should be rotated, it is advisable to mount models on an articulator.

\section{6 - 2 - In the area of the maxillary second premolar}

Orthodontists should incorporate buccal root torque into their appliances in order to facilitate proper occlusion of the palatal cusp of the upper second premolar with the central fossa of the mandibular first molar (fig. 7).

The disparity between bucco-lingual widths of the maxillary second premolar and the mandibular first molar, which occlude with each other, remains the most important problem posed by establishment of a therapeutic Class III occlusion. To compensate for this difference, the orthodontist can deepen the buccal groove of the lower first molar (fig. 9) so it will be better adapted to receive the buccal cusp of the upper second premolar. 


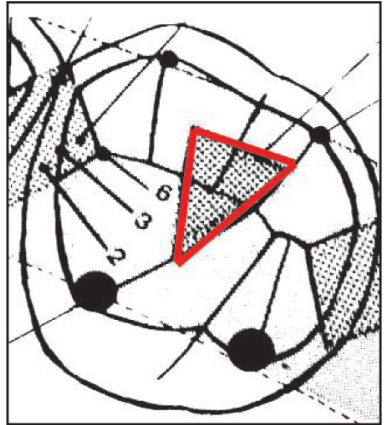

Figure 5

Central fossa of the first maxillary molar. Afger Crétot ${ }^{2}$.

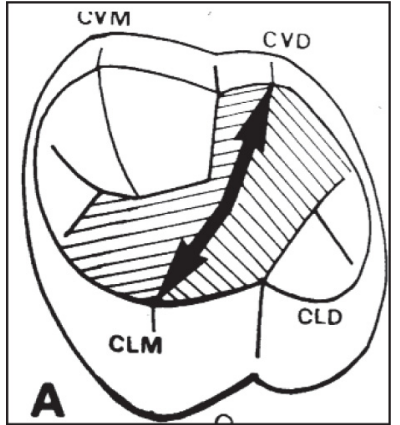

Figure 6

Enamel bridge of the first maxillary molar. After Crétot.

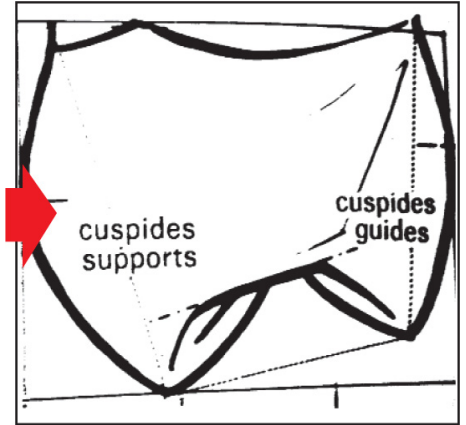

Figure 7

Buccal root torque applied to first maxillary molar. After Crétot.

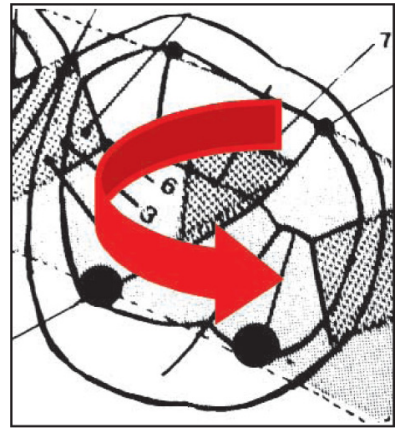

Figure 8 Rotational force to apply to first maxillary molar. After Crétot?.

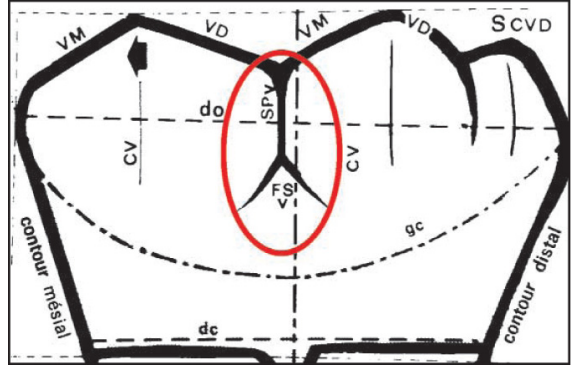

Figure 9

Buccal view of 36: buccal groove. After Crétot2

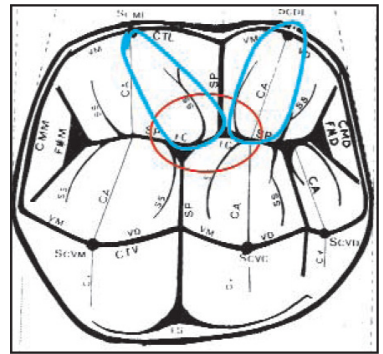

Figure 11

Central fossa of the mandibular first molar (in red) and stabilizing stops (in blue). After Crétot ${ }^{2}$.

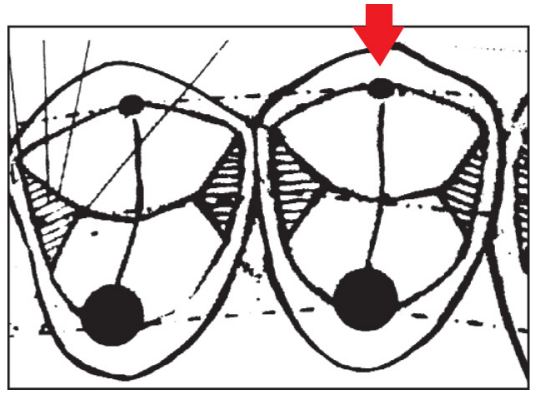

Figure 10

Lingual crown torque to be applied to upper second premolar. After Crétot?

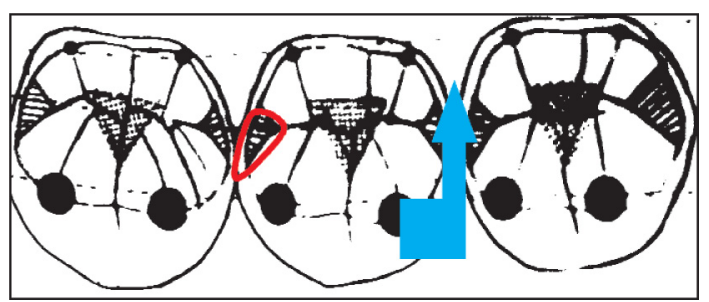

Figure 12

Mesial facet of the mandibular second molar (in red) and the toe-in force to be applied to it (in blue). After Crétot ${ }^{2}$. 


\section{$6-3$ - In the area of the mandibular first molar}

The orthodontist can deepen the small central fossa of the mandibular first molar (fig. 11) with a cylindrical diamond bur and create stops by placing composites on the mesio and disto-lingual cusps of that tooth in order to stabilize its occlusion with the maxillary second premolar.

\section{7 - CRITIQUES}

In orthodontic cases treated to a final therapeutic Class III molar relationship, the lower third molars should have a morphology that allows them to articulate well with the upper second molars. But the question is can one type of molar become an acceptable substitute for another? Lower third molars are often smaller than second molars and are extremely variable in size and shape. Unfortunately, at the beginning of treatment these teeth are usually nothing more than buds, incompletely calcified, and located deep in the body of the mandible. So orthodontists can evaluate them only through radiographs that frequently give an inaccurate picture of these incompletely formed and unerupted teeth, especially if they are malpositioned. This uncertainty may be greatly reduced in the future

\section{6- 4 - In the area of the mandibular second molar}

The orthodontist should deepen the mesial fossa of the mandibular second molar so that the large mesio-palatal cusp of the maxillary first molar will find enough room for proper engagement in occlusion (fig. 12).

It is advisable to apply toe-in force to the lower second molars to improve their occlusion with the upper first molars (fig. 12).

thanks to the introduction of new $\mathrm{X}$-Ray techniques such as NewTom $3 \mathrm{G}$ and Cone Beam CT.

Another issue is that a therapeutic Class III relationship puts the survival of the upper third molars at risk. In that occlusion the only possible occlusal contact that can be envisioned for them is between their mesial margin and the disto-lingual cusps of the lower third molars.

Comparably, the same observation can be made about the lower third molars in cases of therapeutic Class II molar relationships.

Orthodontists must consider the possibility that the congenital absence of lower third molars may constitute a contra-indication for a treatment plan that proposes a finished result in a therapeutic Class III relationship. 


\section{8 - CLINICAL CASE}

The lower second premolars of this patient, a 13 year-old girl (fig. 13 to 16) were congenitally absent. The treatment plan called for the extraction of the lower second temporary molars followed by the mesializing of the lower first molars (fig. 17 to 20).

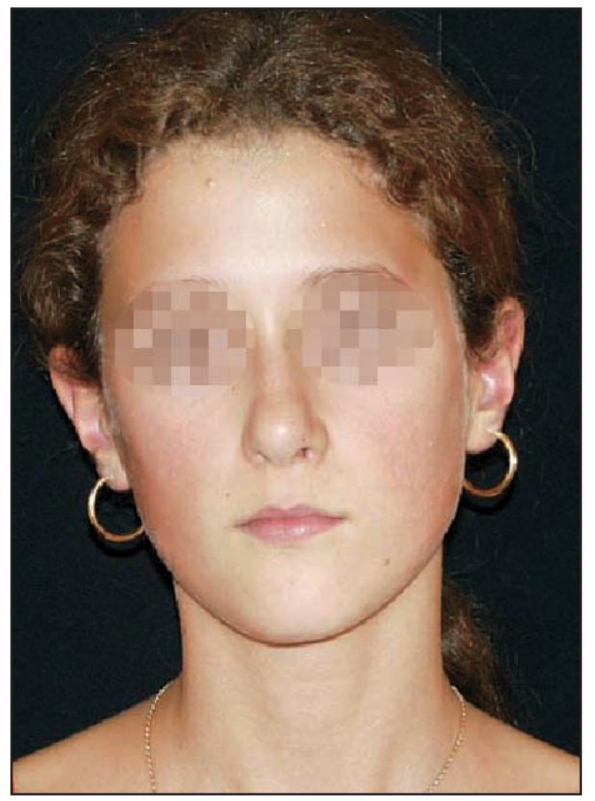

a b

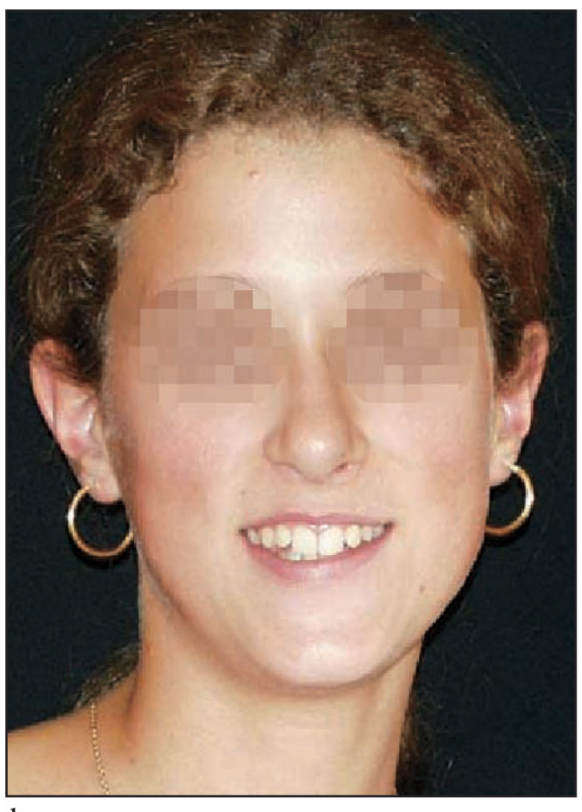

Figures 13 a to $c$

Clinical exam, before treatment.

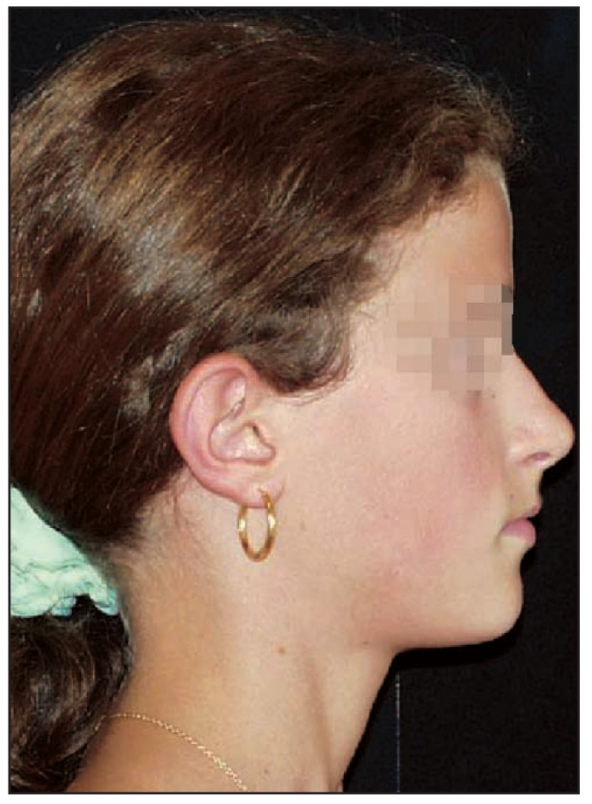

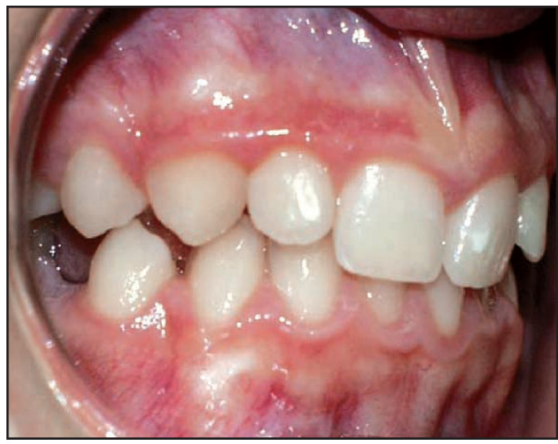

a

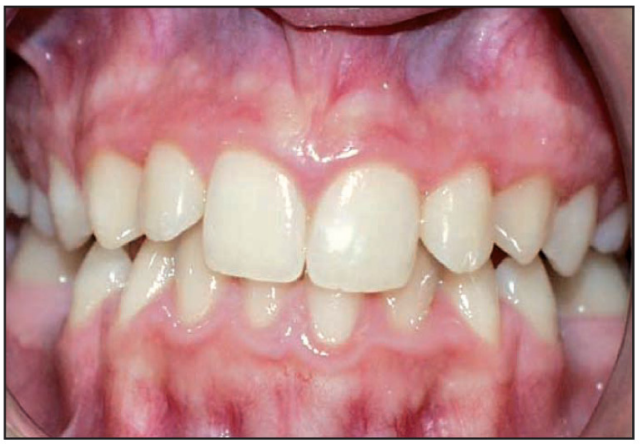

b

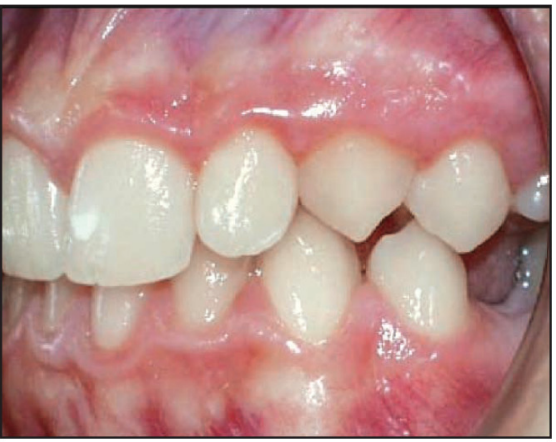

c

Figures 14 a to $c$

Intra-oral photographs, before treatment. 

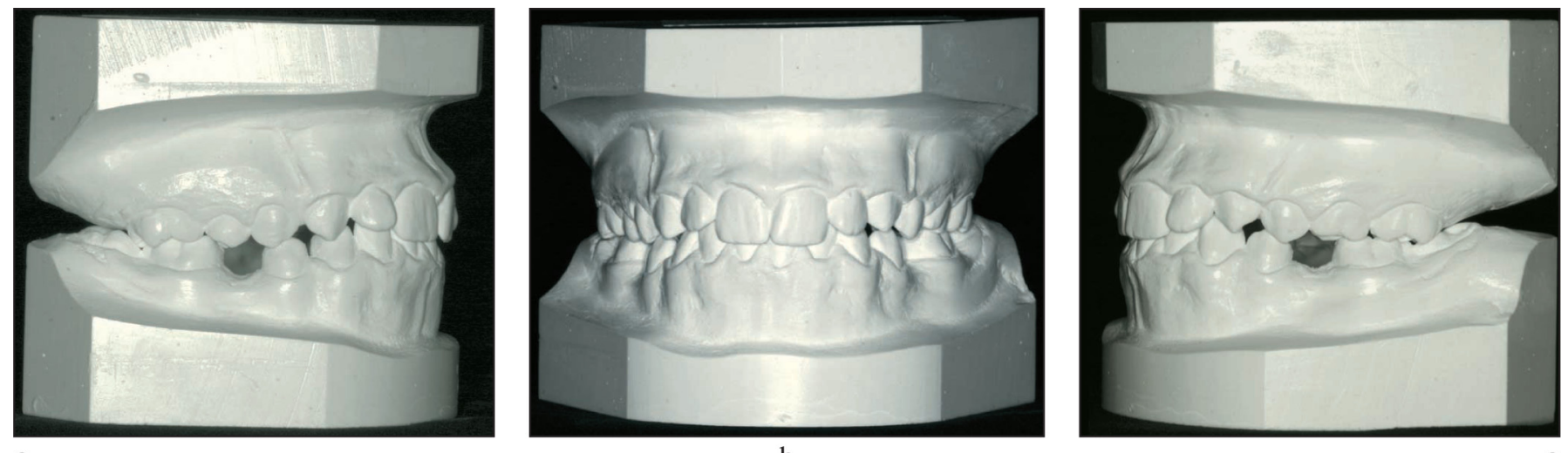

$\mathrm{a}$

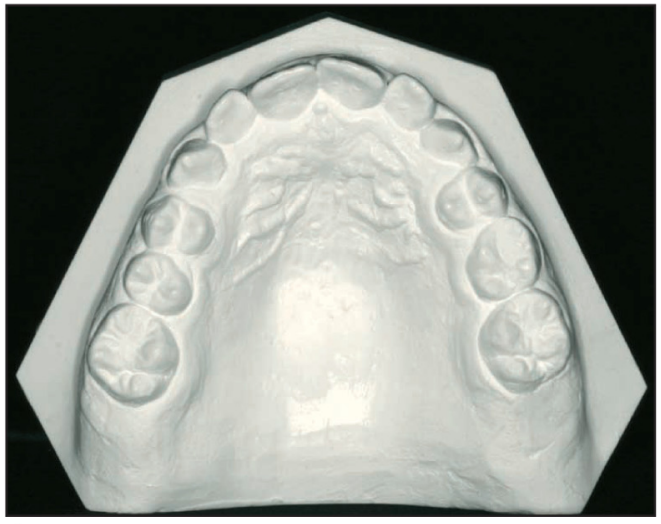

b

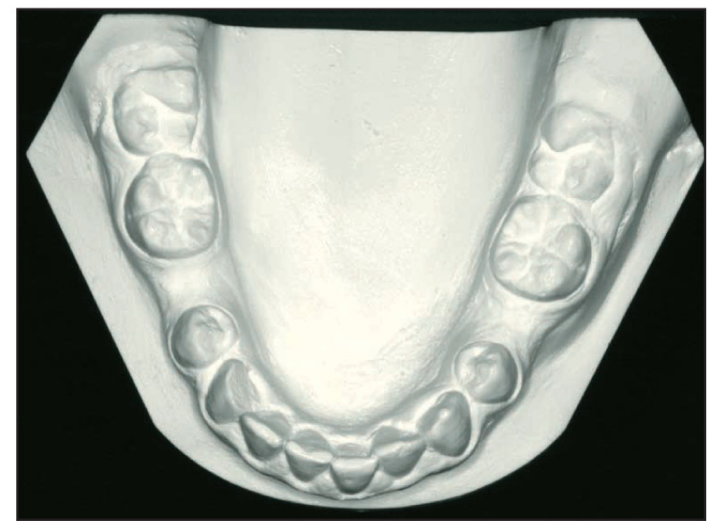

Figures 15 a to e

Supplementary examination, before treatment.
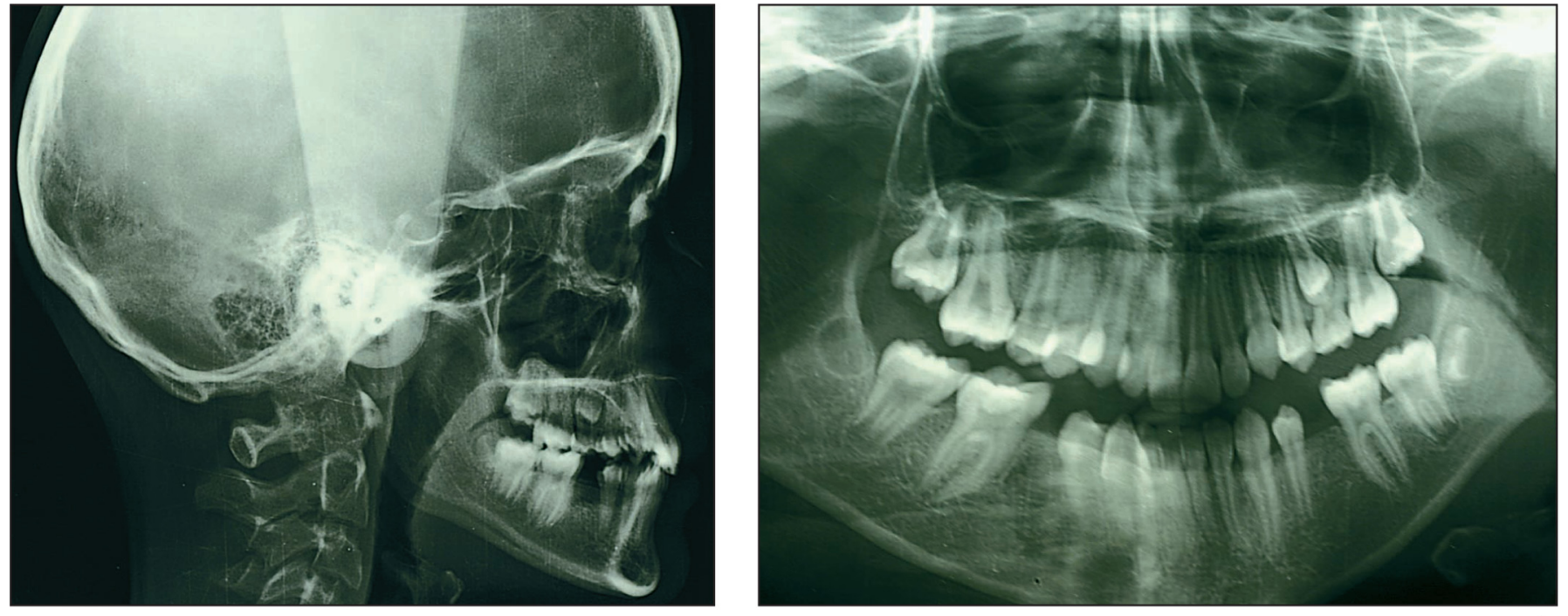

Figures 16 and $b$

Supplementary examination, before treatment. 


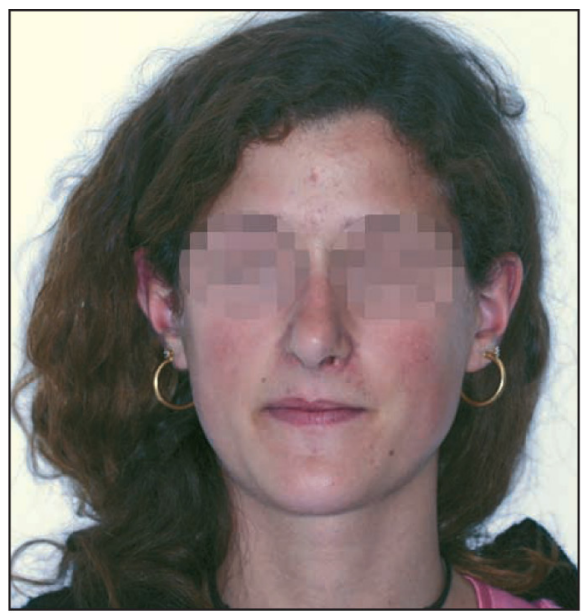

a

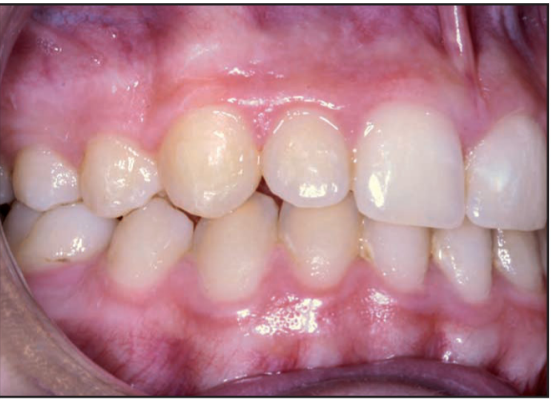

a

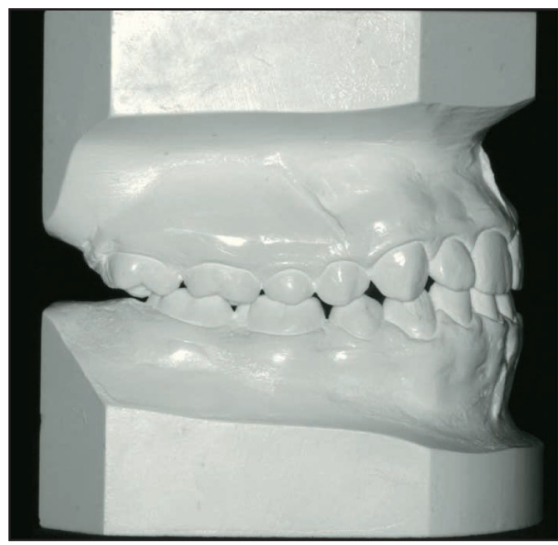

a

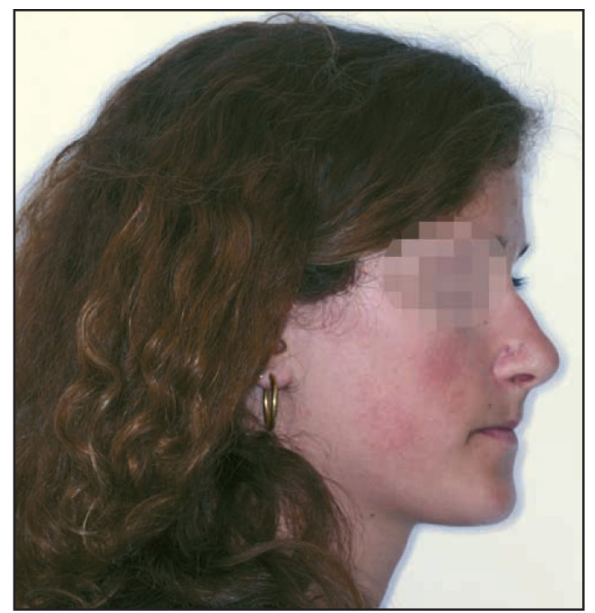

Figures 17 a to $c$

Clinical exam, after treatment.

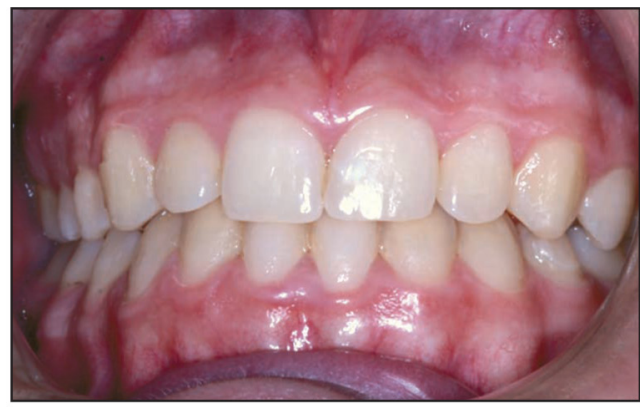

Figures 18 a to $c$

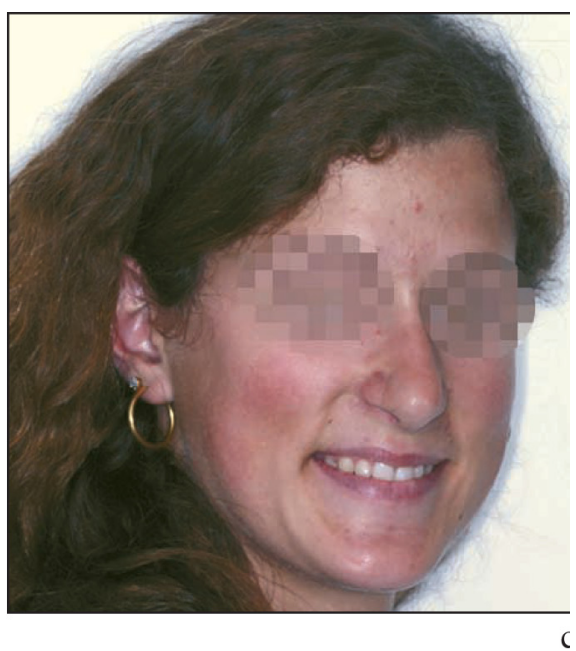

b

Intra-oral photographs, after treatment.

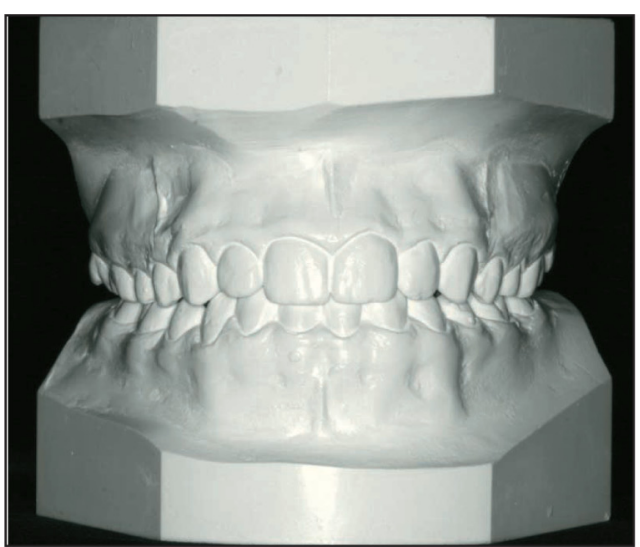

b

Figures $19 a$ and $b$

Supplementary examination, after treatment.

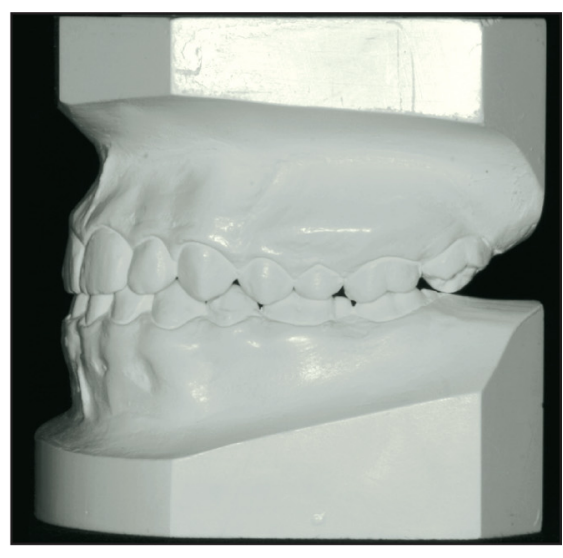

$\mathrm{c}$

Cas des Dr Bachet, Dameron et Le Gall. 

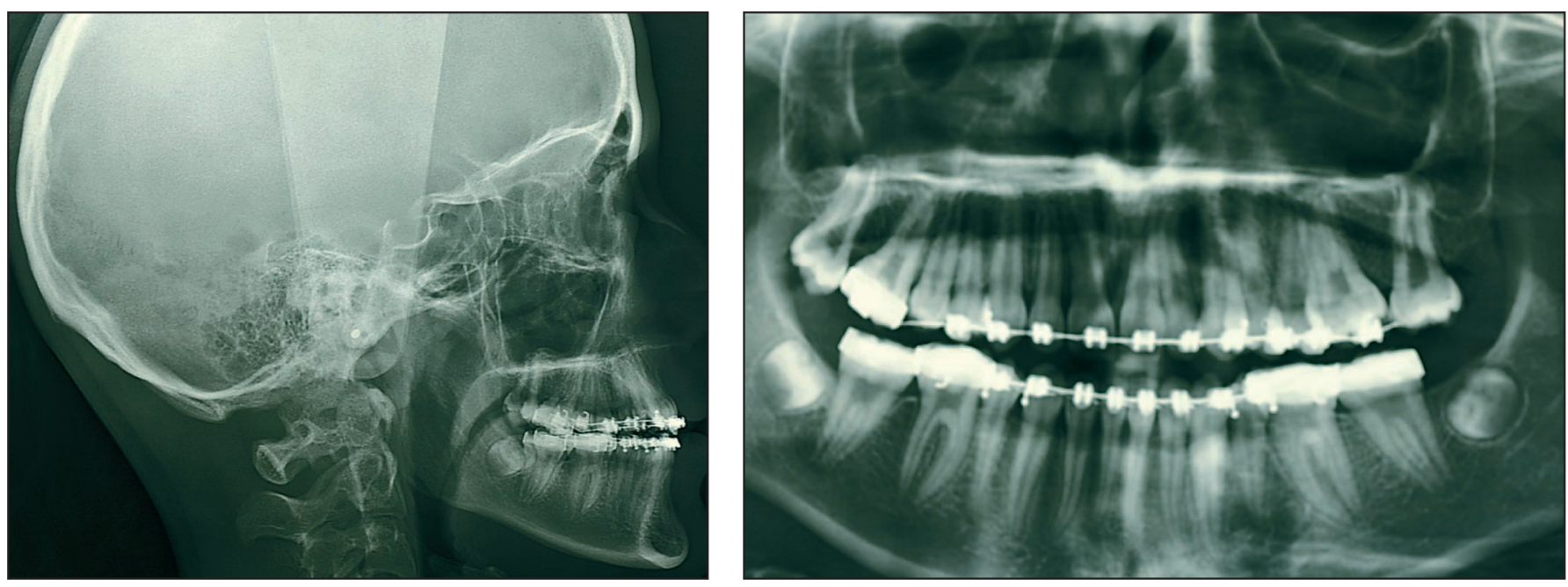

a

Figures 20 and $b$

Supplementary examination, before treatment.

\section{9 - CONCLUSION}

Orthodontists should not reject out of hand a treatment plan that will result in a "therapeutic Class III molar relationship" despite the problems that we have indicated can accompany it because it is an acceptable and, perhaps, preferable solution in certain specific cases.

They should not consider it an easy way out because its proper execution requires very precise adjustments to palliate the natural imperfections that are inherent in it.

Talked about for years but very rarely actually studied, this type of occlusion can be acceptable if the practitioner finishes treatment with a "good" occlusal equilibration. Whenever orthodontists conclude that this therapeutic route might be best for a patient, they should always include a set-up as a part of their diagnostic procedures because it is the only way they can accurately predict the final occlusal result.

If they can put their patient's teeth in a Class III molar relationship that is non-traumatic they can consider it to be a "therapeutic occlusion" provided that the canines are in Class I and the upper and lower incisors function well with each other.

They should also attempt to evaluate the long-term stability of such a result, even if, at this time, not enough evidence is available to provide an answer to this question. 


\section{REFERENCES}

1. Clark JR., Evans RD. Functional occlusion. I. A review. J Orthod 2001;28(1):76-81.

2. Crétot M. L'arcade dentaire humaine. Paris: Editions CdP, 1983.

3 Orthlieb J.D. Occlusion et dysfonction: le paradoxe de l'orthopédie-dento-faciale (Rapport) 1998;69(1).

Michel Le Gall, Maitre de conférences des Universités, chef de service, faculté d'odontologie d'Aix-Marseille II, 27, boulevard Jean-Moulin, 13355 Marseille cedex 5, France.

Danielle DEROZE, Maitre de conférences des Universités, faculté d'odontologie d'Aix-Marseille II, 27, boulevard Jean-Moulin, 13355 Marseille cedex 5, France.

Jean-Daniel Orthlieb, Professeur des Universités, Faculté d'odontologie d'AixMarseille II, 27, boulevard Jean-Moulin, 13355 Marseille cedex 5, France.

Pierre Canal, Professeur des Universités, Faculté d'odontologie de Montpellier, 545, avenue du Professeur Jean-Louis Viala, 34000 Montpellier, France. 